Proceedings of International Mathematical Sciences

ISSN:2717-6355, URL:HTTPS://DERGIPARK.ORG.TR/TR/PUB/PIMS

Volume III Issue 2 (2021), PAges 50-69.

DOI: HTTPS://DOI.ORG/10.47086/PIMS.975424

\title{
A UNIFORMLY STABLE SOLVABILITY OF NLBVP FOR PARAMETERIZED ODE
}

\author{
DOVLET DOVLETOV \\ NEAR EAST UNIVERSITY, MERSIN 10, TURKEY. ORCID NUMBER:0000-0001-9052-8359
}

\begin{abstract}
Nonlocal boundary value problem of the first kind for an ordinary linear second order differential equation with positive parameter at the highest derivative is considered. The existence and uniqueness, as well as, a uniformly stable estimate of classical solution is established under accurate condition on coefficients and location of nonlocal data carriers of multipoint boundary value condition. An essentiality of the revealed condition is confirmed by ill-posed problem examples.
\end{abstract}

\section{INTRODUCTION}

The article of A.N. Tikhonov [1] gave the reason for a wide range study in the field of parameterized differential equations. The joint paper of A.V. Bitsadze and A.A. Samarskii [2] motivated a lot of research in the field of differential problems which are identifiable as nonlocal boundary problems.

In our paper, we consider nonlocal boundary value problem (NLBVP) of the first kind ${ }^{1}$ for ordinary differential equation (ODE)

$$
\varepsilon u^{\prime \prime}(x)+a(x) u^{\prime}(x)-b(x) u(x)=-f(x), \quad 0<x<1
$$

with a positive parameter $\varepsilon>0$. Herein, for an unknown solution, we consider the nonlocal boundary value condition (NLBVC) which is given by linear combination of the values in boundary and interior points of $[0,1]$. Our task is to study the question of a uniformly stable solvability of such NLBVP in respect of the classical solution from $C^{2}(0,1) \cap C[0,1]$.

In 3 , for Sturm-Liouville operator the NLBVP of the first kind

$$
\left[k(x) u^{\prime}\right]^{\prime}-q(x) u=-f(x), 0<x<1, u(0)=0, u(1)=\sum_{i=1}^{n} \alpha_{i} u\left(\xi_{i}\right)
$$

was researched for $k(x) \in C^{1}[0,1], f(x), q(x) \in C[0,1], k(x) \geq m_{0}>0, q(x) \geq 0$. The existence, uniqueness and a priori estimate of classical solution was established

2020 Mathematics Subject Classification. Primary: 35J05; Secondaries: 35A09, 34B10, 65 N06.

Key words and phrases. Poisson's operator, nonlocal boundary value problem, integral kind of nonlocal condition.

C) 2021 Proceedings of International Mathematical Sciences.

Submitted on 28.07.2021 Published on 30.12.2021.

Communicated by Allaberen ASHYRALYEV, and Charyar ASHYRALYYEV.

${ }^{1}$ The term "NLBVP of the first kind" was introduced by V.A. Il'in and E.I. Moiseev in 3. 
for the case if all coefficients $\alpha_{i}, i=1, \ldots, n$ have the same sign and satisfy the condition

$$
-\infty<\sum_{i=1}^{n} \alpha_{i} \leq 1
$$

For the same problem, but under the condition that $\alpha_{i}, i=1, \ldots, n$ have an arbitrary sign and satisfy the condition

$$
\sum_{i=1}^{n} \frac{\left(\alpha_{i}+\left|\alpha_{i}\right|\right)}{2} \int_{0}^{\xi_{i}} \frac{1}{k(\tau)} d \tau<\int_{0}^{1} \frac{1}{k(\tau)} d \tau
$$

the existence and uniqueness of classical solution was proved in 4 .

In [5], it was proved that singularly perturbed NLBVP of the first kind

$$
-\varepsilon^{2} y^{\prime \prime}(x)+g(x) y(x)=h(x), \quad 0<x<1, \quad y(0)=0, \hat{\ell} y=d,
$$

has a unique solution if and only if the solution of Dirichlet problem

$$
\varepsilon^{2} u^{\prime \prime}(x)-g(x) u(x)=0, u(0)=0, u(1)=1
$$

satisfies the condition $\hat{\ell} u \neq 0$, where $g(x) \geq K^{2}>0, K \in \boldsymbol{R}, \hat{\ell} y \equiv y(1)-\sum_{i=1}^{m} c_{i} y\left(s_{i}\right)$, $s_{i} \in(0,1)$.

In [6], the existence, uniqueness and a priori estimate of classical solution

$$
\|u\|_{W_{2}^{2}[0,1]} \leq C\|f\|_{L_{2}[0,1]}
$$

were proved for NLBVP

$$
\left\{\begin{array}{l}
{\left[k(x) u^{\prime}(x)\right]^{\prime}+r(x) u^{\prime}(x)-q(x) u(x)=-f(x), \quad 0<x<1,} \\
u(0)=0, u(1)=\alpha u(\zeta)-\beta u(\eta),
\end{array}\right.
$$

where $k(x) \in C^{1}[0,1], f(x), r(x)$ and $q(x) \in C[0,1], k(x) \geq m_{0}>0,|r(x)|<\mu$, $q(x) \geq 0, \quad x \in[0,1], \quad \mu<m_{0}, \quad \zeta \in(0,1), \quad \eta \in(0,1)$, in addition, $\alpha>0, \beta>0$, $-\infty<\alpha-\beta \leq 1$ if $\zeta<\eta, \alpha \leq 1$ if $\eta<\zeta$.

In [7], the existence, uniqueness and a priori estimate of classical solution were proved for NLBVP with double-side NLBVC of the first kind

$$
\left\{\begin{array}{l}
{\left[k(x) u^{\prime}(x)\right]^{\prime}+r(x) u^{\prime}(x)-q(x) u(x)=-f(x), \quad 0<x<1,} \\
u(0)=\alpha_{0} u\left(\zeta_{0}\right)-\beta_{0} u\left(\eta_{0}\right), u(1)=\alpha_{1} u\left(\zeta_{1}\right)-\beta_{1} u\left(\eta_{1}\right)
\end{array}\right.
$$

where $k(x) \in C^{1}[0,1], \quad f(x), r(x), q(x) \in C[0,1], k(x) \geq m_{0}>0, q(x) \geq 0$, $x \in[0,1], \zeta_{i} \in(0,1), \eta_{i} \in(0,1), i=0,1, \max \left\{\zeta_{0}, \eta_{0}\right\}<\min \left\{\zeta_{1}, \eta_{1}\right\}$, in addition, $\alpha_{i}>0, \beta_{i}>0, i=0,1, S_{0} \leq 1, S_{1} \leq 1, S_{0}+S_{1}<2$, herewith $S_{0}=\alpha_{0}-\beta_{0}$ for $\eta_{0} \leq \zeta_{0}, S_{0}=\alpha_{0}$ for $\zeta_{0}<\eta_{0}, S_{1}=\alpha_{1}-\beta_{1}$ for $\zeta_{1} \leq \eta_{1}, S_{1}=\alpha_{1}$ for $\eta_{1}<\zeta_{1}$.

In [8, p. 68-72], a uniformly stable solvability was reported for parameterized NLBVP

$$
\left\{\begin{array}{l}
-\varepsilon u^{\prime \prime}(x)+b(x) u(x)=f(x), \quad 0<x<1, \\
u(0)-\alpha u(\zeta)=\phi_{0}, \quad u(1)-\beta u(\eta)=\phi_{1}
\end{array}\right.
$$

where $\varepsilon>0, b(x), f(x) \in C[0,1], b(x) \geq b^{*}>0,0<\zeta<\eta<1,-\infty<\alpha<1$, $-\infty<\beta<1, \alpha \beta \neq 0, \varphi_{i}=$ const, $i=0,1$.

In [9], the solution of NLBVP, which was formulated in [5], was constructed by using the truncated orthogonal series and corresponding solution of the reduced problem. 
In [10], under the condition that classical solution of the Dirichlet problem

$$
-\varepsilon w^{\prime \prime}+a(x) w^{\prime}+b(x) w=0,0<x<1, w(0)=0, w(1)=1
$$

satisfies the inequality $w(1)-\sum_{i=1}^{m-2} c_{i} w\left(s_{i}\right) \neq 0$, the behaviour of exact solution was analized for NLBVP

$$
-\varepsilon u^{\prime \prime}+a(x) u^{\prime}+b(x) u=f(x), 0<x<1, u(0)=A, u(1)=\sum_{i=1}^{m-2} c_{i} u\left(s_{i}\right)+B,
$$

where $0<\varepsilon<<1, a(x) \geq \alpha>0, a(x), b(x)$ and $f(x)$ are sufficiently smooth functions on $[0,1], s_{i} \in(0,1), i=1,2, \ldots, m-2$.

In summary, it is natural that NLBVP's solvability, as well as, the behaviour of its classical solution depends on coefficients, their signs, values, and, at least, data carriers location of given nonlocal condition. It is the reason why the aim of our paper is to reveal explicit condition of a uniform solvability for parameterized linear second order ODE with abstract double-side nonlocal condition of the first kind. In general, naturally that the information on a uniform solvability of differential problem is also actual for its numerical interpretation.

Additionally, sufficiently detailed overview on NLBVP for ODE is enclosed in [3, 4, 6, 7, 13, the survey on boundary value problems respectively parameterized ODE is represented by [14].

\section{Differential Problem}

We consider the NLBVP

$$
\begin{array}{r}
L u(x) \equiv \varepsilon u^{\prime \prime}(x)+a(x) u^{\prime}(x)-b(x) u(x)=-f(x), \quad 0<x<1, \\
\ell_{0}(u) \equiv u(0)-\sum_{k=1}^{m_{0}} \alpha_{k} u\left(\zeta_{k}\right)=\varphi_{0}, \quad \ell_{1}(u) \equiv u(1)-\sum_{l=1}^{m_{1}} \beta_{l} u\left(\eta_{l}\right)=\varphi_{1},
\end{array}
$$

where $\varepsilon>0$ is a parameter, $a(x), \quad b(x), \quad f(x) \in C[0,1], \quad m_{i} \geq 2, \quad i=0,1$, $\varphi_{i} \in R, i=0,1, \zeta_{k} \in(0,1), k=1, \ldots, m_{0}, \eta_{l} \in(0,1), l=1, \ldots, m_{1}$ are so that

$$
0<\zeta_{1}<\zeta_{2}<\ldots<\zeta_{m_{0}}<\eta_{1}<\eta_{2}<\ldots<\eta_{m_{1}}<1
$$

in addition, $\alpha_{k} \in R, k=1, \ldots, m_{0}, \beta_{l} \in R, l=1, \ldots, m_{1}$ are nonzero coefficients. Next condition is denoted by $\boldsymbol{A}$ :

- if all $\alpha_{k}$ are not of the same sign, then $\alpha_{k}>0$ only for $k=1, \ldots, m_{\iota}$, or $\alpha_{k}>0$ only for $k=m_{\iota}+1, \ldots, m_{0}$, where $m_{\iota}$ is some natural number, $1 \leq m_{\iota}<m_{0}$;

- if all $\beta_{l}$ are not of the same sign, then $\beta_{l}>0$ only for $l=1, \ldots, m_{\kappa}$, or $\beta_{l}>0$ only for $l=m_{\kappa}+1, \ldots, m_{1}$, where $m_{\kappa}$ is some natural number, $1 \leq m_{\kappa}<m_{1}$. Further, we will use the designations:

$$
\begin{gathered}
\alpha=\sum_{k=1}^{m_{0}} \alpha_{k}, \quad \alpha^{+}=\sum_{k=1}^{m_{0}} \frac{\alpha_{k}+\left|\alpha_{k}\right|}{2}, \quad \alpha^{-}=\sum_{k=1}^{m_{0}} \frac{\alpha_{k}-\left|\alpha_{k}\right|}{2}, \\
\beta=\sum_{l=1}^{m_{1}} \beta_{l}, \quad \beta^{+}=\sum_{l=1}^{m_{1}} \frac{\beta_{l}+\left|\beta_{l}\right|}{2}, \beta^{-}=\sum_{l=1}^{m_{1}} \frac{\beta_{l}-\left|\beta_{l}\right|}{2}, \\
S_{0}=\left\{\begin{array}{l}
\alpha^{+}+\alpha^{-}, \text {if } \alpha_{m_{\iota}}<0, \alpha_{m_{l+1}}>0, \\
\alpha^{+}, \text {if } \alpha_{m_{\iota}}>0, \alpha_{m_{\iota+1}}<0, \\
\alpha, \text { if } \alpha_{k}, k=1, \ldots, m_{0} \text { have the same sign },
\end{array}\right.
\end{gathered}
$$




$$
S_{1}=\left\{\begin{array}{l}
\beta^{+}+\beta^{-}, \text {if } \beta_{m_{\kappa}}>0, \beta_{m_{\kappa}+1}<0 \\
\beta^{+}, \text {if } \beta_{m_{\kappa}}<0, \beta_{m_{\kappa+1}}>0 \\
\beta, \text { if } \beta_{l}, l=1, \ldots, m_{1} \text { have the same sign }
\end{array}\right.
$$

Definition. The function $u(x)$ is a classical solution of NLBVP (2.1)-(2.2) if it belongs to $C^{2}(0,1) \cap C[0,1]$, satisfies the equation (2.1) and NLBVC (2.2).

Let each one of NLBVC (2.2) encloses different sign coefficients. Let us suppose that classical solution $u(x)$ of NLBVP (2.1)-(2.2) exists. Then, in view of the mean value (MV) property [3, p. 1198-1199], by analogy with [13, p. 39], this classical solution satisfies some reduced NLBVC

$$
u(0)-\alpha^{+} u\left(\zeta^{+}\right)-\alpha^{-} u\left(\zeta^{-}\right)=\varphi_{0}, \quad u(1)-\beta^{+} u\left(\eta^{+}\right)-\beta^{-} u\left(\eta^{-}\right)=\varphi_{1},
$$

where $\zeta^{+} \in\left[\zeta_{1}, \zeta_{m_{0}}\right], \zeta^{-} \in\left[\zeta_{1}, \zeta_{m_{0}}\right], \eta^{+} \in\left[\eta_{1}, \eta_{m_{1}}\right], \eta^{-} \in\left[\eta_{1}, \eta_{m_{1}}\right]$ and, therefore, $u(x)$ is classical solution of NLBVP $(2.1),(2.4)$ tod $^{2}$ In respect of (2.4), we denote

$$
\bar{\ell}_{0}(u) \equiv u(0)-\alpha^{+} u\left(\zeta^{+}\right)-\alpha^{-} u\left(\zeta^{-}\right), \quad \bar{\ell}_{1}(u) \equiv u(1)-\beta^{+} u\left(\eta^{+}\right)-\beta^{-} u\left(\eta^{-}\right) .
$$

Hence, for $S_{i}, i=0,1$, we have

$$
\begin{aligned}
& S_{0}=\left\{\begin{array}{l}
\alpha^{+}+\alpha^{-}, \text {if } \zeta^{-}<\zeta^{+}, \\
\alpha^{+}, \text {if } \zeta^{+}<\zeta^{-}, \\
\alpha, \text { if all } \alpha_{k}, k=1, \ldots, m_{0} \text { have the same sign, }
\end{array}\right. \\
& S_{1}=\left\{\begin{array}{l}
\beta^{+}+\beta^{-}, \text {if } \eta^{+}<\eta^{-}, \\
\beta^{+}, \text {if } \eta^{-}<\eta^{+}, \\
\beta, \text { if all } \beta_{l}, l=1, \ldots, m_{1} \text { have the same sign. }
\end{array}\right.
\end{aligned}
$$

Additionally, in view of (2.3) and $\boldsymbol{A}$, we have

$$
\zeta^{-} \neq \zeta^{+}, \quad \eta^{-} \neq \eta^{+}, \max \left\{\zeta^{-}, \zeta^{+}\right\}<\min \left\{\eta^{-}, \eta^{+}\right\} .
$$

Our first result is

Lemma 2.1. Let $S_{i} \leq 1, \varphi_{i} \neq 0, i=0,1$. If $u(x)$ is classical solution of the problem (2.1),(2.4), then $v(x)=u(x)+\varphi_{0} q_{0}(x)+\varphi_{1} q_{1}(x)$ is classical solution of the problem

$$
L v(x)=-f_{1}(x), 0<x<1, \quad \bar{\ell}_{0}(v)=0, \quad \bar{\ell}_{1}(v)=0
$$

for $f_{1}(x)=f(x)-\varphi_{0} L q_{0}(x)-\varphi_{1} L q_{1}(x)$, where $q_{i}(x), \quad i=0,1$ are some cubic polinoms .

Let $S_{i} \leq 1, i=0,1$. Let only one of $\varphi_{0}, \varphi_{1}$ be nonzero, i.e., $\varphi_{i_{*}} \neq 0, i_{*} \in\{0,1\}$. If $u(x)$ is classical solution of (2.1),(2.4), then $v(x)=u(x)+\varphi_{i_{*}} q_{i_{*}}(x)$ is classical solution of the problem (2.9) for $f_{1}(x)=f(x)-\varphi_{i_{*}} L q_{i_{*}}(x)$, where $q_{i_{*}}(x)$ is some cubic polinom.

Proof. Assume that $q_{0}(x), q_{1}(x) \in C^{2}(0,1)$ are an arbitrary functions. Then it is obvious that

$$
L v(x)=-\left[f(x)-\varphi_{0} L q_{0}(x)-\varphi_{1} L q_{1}(x)\right]=-f_{1}(x)
$$

\footnotetext{
${ }^{2}$ Thus we will say: "the problem $(2.1),(2.2)$ is reduciable to $(2.1),(2.4)$ ", or, for example, "condition (2.2) is redused to (2.4)", or "condition (2.2) is reduciable to (2.4)", or "(2.4) is reduced nonlocal condition" and etc..
} 
i.e., $v(x)$ satisfies the differential equation (2.9). Let us construct some polinomials $q_{0}(x)$ and $q_{1}(x)$, so that the function $v(x)$ will satisfy $\operatorname{NLBVC~(2.9).~Put~} \varphi_{i} \neq 0$, $i=0,1$. We look for the functions

$$
\begin{gathered}
q_{0}(x)=c_{0}(1-x)\left(\eta^{+}-x\right)\left(\eta^{-}-x\right), \\
q_{1}(x)=c_{1} x\left(\zeta^{+}-x\right)\left(\zeta^{-}-x\right),
\end{gathered}
$$

where an unknown constants $c_{0}$ and $c_{1}$ have to be defined. Since

$$
\bar{\ell}_{0}\left(q_{1}\right)=0, \quad \bar{\ell}_{1}\left(q_{0}\right)=0,
$$

then, in view of (2.5) and (2.4),

$$
\begin{aligned}
& \bar{\ell}_{0}(v)=\varphi_{0}+\varphi_{0} \bar{\ell}_{0}\left(q_{0}\right)+\varphi_{1} \bar{\ell}_{0}\left(q_{1}\right)=\varphi_{0}\left[1+\bar{\ell}_{0}\left(q_{0}\right)\right], \\
& \bar{\ell}_{1}(v)=\varphi_{1}+\varphi_{0} \bar{\ell}_{1}\left(q_{0}\right)+\varphi_{1} \bar{\ell}_{1}\left(q_{1}\right)=\varphi_{1}\left[1+\bar{\ell}_{1}\left(q_{1}\right)\right] .
\end{aligned}
$$

Since $v(x)$ has to satify (2.9), then, in view of (2.13) and (2.14), the equalities

$$
1+\bar{\ell}_{0}\left(q_{0}\right)=0, \quad 1+\bar{\ell}_{1}\left(q_{1}\right)=0
$$

have to be satisfied for $q_{i}(x), i=0,1$. Hence, we have

$$
\begin{aligned}
& c_{0}=-\left(E_{0}\right)^{-1}, \\
& c_{1}=-\left(D_{0}\right)^{-1},
\end{aligned}
$$

for

$$
\begin{aligned}
& E_{0}=\eta^{+} \eta^{-}-\alpha^{+}\left(1-\zeta^{+}\right)\left(\eta^{+}-\zeta^{+}\right)\left(\eta^{-}-\zeta^{+}\right)-\alpha^{-}\left(1-\zeta^{-}\right)\left(\eta^{+}-\zeta^{-}\right)\left(\eta^{-}-\zeta^{-}\right), \\
& D_{0}=\left(1-\zeta^{+}\right)\left(1-\zeta^{-}\right)-\beta^{+} \eta^{+}\left(\zeta^{+}-\eta^{+}\right)\left(\zeta^{-}-\eta^{+}\right)-\beta^{-} \eta^{-}\left(\zeta^{+}-\eta^{-}\right)\left(\zeta^{-}-\eta^{-}\right),
\end{aligned}
$$

where $E_{0} \neq 0, D_{0} \neq 0$ since $E_{0}>0, D_{0}>0$. Indeed, in view of (2.8), from (2.18) and (2.19), correspondingly, we get

$$
\begin{gathered}
E_{0}>\left\{\begin{array}{l}
\left(1-\left[\alpha^{+}+\alpha^{-}\right]\right)\left(1-\zeta^{-}\right)\left(\eta^{+}-\zeta^{-}\right)\left(\eta^{-}-\zeta^{-}\right), \text {if } \zeta^{-}<\zeta^{+}, \\
\left(1-\alpha^{+}\right)\left(1-\zeta^{-}\right)\left(\eta^{+}-\zeta^{-}\right)\left(\eta^{-}-\zeta^{-}\right), \text {if } \zeta^{+}<\zeta^{-},
\end{array}\right. \\
D_{0}>\left\{\begin{array}{l}
\left(1-\left[\beta^{+}+\beta^{-}\right]\right) \eta^{-}\left(\eta^{-}-\zeta^{+}\right)\left(\eta^{-}-\zeta^{-}\right), \text {if } \eta^{+}<\eta^{-}, \\
\left(1-\beta^{+}\right) \eta^{-}\left(\eta^{-}-\zeta^{+}\right)\left(\eta^{-}-\zeta^{-}\right), \text {if } \eta^{-}<\eta^{+} .
\end{array}\right.
\end{gathered}
$$

Then from (2.20) and (2.21), correspondingly, in view of (2.6) and (2.7), we have

$$
\begin{gathered}
E_{0}>\left\{\begin{array}{l}
\left(1-S_{0}\right)\left(1-\zeta^{-}\right)\left(\eta^{+}-\zeta^{-}\right)\left(\eta^{-}-\zeta^{-}\right), \text {if } \zeta^{-}<\zeta^{+}, 0<S_{0} \leq 1, \\
\left(1-\zeta^{-}\right)\left(\eta^{+}-\zeta^{-}\right)\left(\eta^{-}-\zeta^{-}\right), \text {if } \zeta^{-}<\zeta^{+},-\infty<S_{0} \leq 0, \\
\left(1-S_{0}\right)\left(1-\zeta^{-}\right)\left(\eta^{+}-\zeta^{-}\right)\left(\eta^{-}-\zeta^{-}\right), \text {if } \zeta^{+}<\zeta^{-}, 0 \leq S_{0} \leq 1,
\end{array}\right. \\
D_{0}>\left\{\begin{array}{l}
\left(1-S_{1}\right) \eta^{-}\left(\eta^{-}-\zeta^{+}\right)\left(\eta^{-}-\zeta^{-}\right), \text {if } \eta^{+}<\eta^{-}, 0<S_{1} \leq 1, \\
\eta^{-}\left(\eta^{-}-\zeta^{+}\right)\left(\eta^{-}-\zeta^{-}\right), \text {if } \eta^{+}<\eta^{-},-\infty<S_{1} \leq 0, \\
\left(1-S_{1}\right) \eta^{-}\left(\eta^{-}-\zeta^{+}\right)\left(\eta^{-}-\zeta^{-}\right), \text {if } \eta^{-}<\eta^{+}, 0 \leq S_{1} \leq 1 .
\end{array}\right.
\end{gathered}
$$

Hence, $E_{0}>0, D_{0}>0$, therefore, $E_{0} \neq 0, D_{0} \neq 0$. Thus, in view of (2.16) and (2.17), the polinomials (2.10) and (2.11) are defined. In view of (2.12) and (2.15), $v(x)$ satisfies nonlocal conditions of $(2.9)$. Since $u(x) \in C^{2}(0,1) \cap C[0,1]$, then $v(x) \in C^{2}(0,1) \cap C[0,1]$ too, therefore, $v(x)$ is classical solution of NLBVP $(2.9)$.

By similar way, it is easy to prove the second statement for the case if one of two data $\varphi_{i}, i=0,1$ is zero, but another one is nonzero. Lemma 2.1 is proved. 
Let all coefficients $\alpha_{k}, \quad k=1, \ldots, m_{0}$ have the same sign and all coefficients $\beta_{l}, l=1, \ldots, m_{1}$ have the same sign, (the signs of $\alpha_{k}$ and $\beta_{l}$ can be different). Then, by analogy with (2.4), the classical solution of NLBVP (2.1),(2.2) satisfies the condition

$$
u(0)-\alpha u(\zeta)=\varphi_{0}, \quad u(1)-\beta u(\eta)=\varphi_{1},
$$

for some $\zeta \in\left[\zeta_{1}, \zeta_{m_{0}}\right], \eta \in\left[\eta_{1}, \eta_{m_{1}}\right]$, so that $\zeta<\eta$ in view of (3).

Corollary 2.2. Let $S_{i} \leq 1, \varphi_{i} \neq 0, i=0,1$. If $u(x)$ is some classical solution of the problem

$$
L u(x)=-f(x), 0<x<1, u(0)-\alpha u(\zeta)=\varphi_{0}, u(1)-\beta u(\eta)=\varphi_{1}
$$

for $\zeta<\eta$, then $v(x)=u(x)+\varphi_{0} q_{0}(x)+\varphi_{1} q_{1}(x)$ is classical solution of the problem

$$
L v(x)=-f_{1}(x), 0<x<1, \quad v(0)-\alpha v(\zeta)=0, v(1)-\beta v(\eta)=0
$$

for $f_{1}(x)=f(x)-\varphi_{0} L q_{0}(x)-\varphi_{1} L q_{1}(x), q_{0}(x)=c_{0}(1-x)(\eta-x), q_{1}(x)=c_{1} x(\zeta-x)$, $c_{0}=-[\eta-\alpha(1-\zeta)(\eta-\zeta)]^{-1}, c_{1}=-[(1-\zeta)-\beta \eta(\eta-\zeta)]^{-1}$.

Proof. This is provable by analogy with Lemma 2.1. Corollary 2.2 is proved.

Corollary 2.3. The statement of Corollary 2.2 is true for the case if all coefficients $\alpha_{k}, k=1, \ldots, m_{0}$ have the same sign, but there are different sign coefficients among $\beta_{l}, l=1, \ldots, m_{1}$ (or vice versa).

Proof. This is provable by analogy with Lemma 2.1. Corollary 2.3 is proved.

\section{A UNIFORM STABILITy ESTIMATE}

Here, we establish a uniformly stable estimate. Our basic result is

Theorem 3.1. Let $a(x) \geq a_{0}>0, b(x) \geq b_{0} \geq 0, x \in[0,1]$. Let conditions (2.3), $\boldsymbol{A}$ hold. If $S_{0} \leq 1, S_{1} \leq 1$ and, in addition, $S_{1}<1$ if $b_{0}=0$, then a uniformly stable estimate

$$
|u(x)| \leq C\left(\left|\varphi_{0}\right|+\left|\varphi_{1}\right|+\max _{0 \leq y \leq 1}|f(y)|\right), \quad 0 \leq x \leq 1
$$

holds for classical solution of NLBVP (2.1),(2.2).

Proof. Let $u(x)$ be some classical solution of NLBVP (2.1),(2.2). Since (2.2) is reduciable to (2.4), then $u(x)$ is classical solution of NLBVP $(2.1),(2.4)$. In view of Lemma 2.1, the function $v(x)=u(x)+\varphi_{0} q_{0}(x)+\varphi_{1} q_{1}(x)$ is classical solution of NLBVP (2.9). Assume that a uniformly stable estimate holds for $v(x)$, i.e.,

$$
|v(x)| \leq C_{1} \max _{0 \leq y \leq 1}\left|f_{1}(y)\right|, \quad 0 \leq x \leq 1
$$

for some independent of $\varepsilon$ constant $C_{1}$, where $f_{1}(x)=f(x)-\varphi_{0} L q_{0}(x)-\varphi_{1} L q_{1}(x)$. Then, by virtue of the triangle inequality,

$$
|u(x)| \leq C_{1} \max _{0 \leq y \leq 1}\left|f_{1}(y)\right|+\left|\varphi_{0}\right| \max _{0 \leq y \leq 1}\left|q_{0}(y)\right|+\left|\varphi_{1}\right| \max _{0 \leq y \leq 1}\left|q_{1}(y)\right|, \quad 0 \leq x \leq 1,
$$

so that

$$
|u(x)| \leq C_{1} \max _{0 \leq y \leq 1}|f(y)|+C_{2}\left|\varphi_{0}\right|+C_{3}\left|\varphi_{1}\right|, \quad 0 \leq x \leq 1 .
$$

Thus, if (3.2) is true, then (3.1) is also true for the constant $C=\max \left\{C_{1}, C_{2}, C_{3}\right\}$. So, to prove (3.1) it will be sufficient to obtain the estimate (3.2) for the solution of NLBVP (2.9). Further, to establish (3.2) we will consider three subcases: 
first - all coefficients $\alpha_{k}, k=1, \ldots, m_{0}$ have the same sign and all coefficients $\beta_{l}$, $l=1, \ldots, m_{1}$ have the same sign (the signs of $\alpha_{k}$ and $\beta_{l}$ can be different);

second - each one of two nonlocal conditions (2.2) encloses different sign coefficients; third - one condition of (2.2) has the same sign coefficients, but another one encloses different sign coefficients.

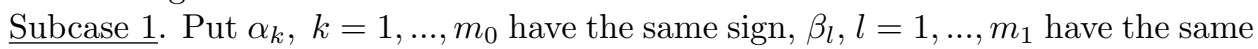
sign (the signs of $\alpha_{k}$ and $\beta_{l}$ can be different). Our task is to prove the estimate (3.2) for classical solution of NLBVP (2.9). By virtue of MV property [3, p. 1198-1199] in respect of NLBVC (2.9), we get

$$
v(0)=\alpha v(\zeta), \quad v(1)=\beta v(\eta)
$$

for some $\zeta \in\left[\zeta_{1}, \zeta_{m_{0}}\right], \eta \in\left[\eta_{1}, \eta_{m_{1}}\right]$. If $\alpha<0$, then, in view of Bolzano theorem, $v\left(x_{0}\right)=0$ at some point $x_{0} \in(0, \zeta)$, i.e., $v(x)$ satisfies boundary value condition (BVC) of the first kind at $x_{0}$. If $\alpha>0$, then $\omega_{0}(0)=\omega_{0}(\zeta)$ for the function

$$
\omega_{0}(x)=v(x) \frac{(\alpha-1) x+\zeta}{\zeta} .
$$

By virtue of Rolle's theorem, $\omega_{0}^{\prime}\left(x_{0}\right)=0$ at some point $x_{0} \in(0, \zeta)$. Hence,

$$
v^{\prime}\left(x_{0}\right)-h_{0} v\left(x_{0}\right)=0, \quad h_{0}=\frac{1-\alpha}{\zeta-x_{0}(1-\alpha)},
$$

so that $h_{0} \geq 0$ since our theorem condition requires the bound $S_{0} \leq 1$. It means that $v(x)$ satisfies BVC of the third kind at $x_{0}$ if $0<\alpha<1$, or of the second kind if $\alpha=1$.

Similarly, for $\beta<0$ we obtain BVC of the first kind $v\left(x_{1}\right)=0$ at some point $x_{1} \in(\eta, 1)$, as well as, for $\beta>0$ we get BVC of the third kind if $0<\beta<1$, or of the second kind if $\beta=1$ at some point $x_{1} \in(\eta, 1)$, i.e.,

$$
v^{\prime}\left(x_{1}\right)+h_{1} v\left(x_{1}\right)=0, \quad h_{1}=\frac{1-\beta}{\beta\left(1-x_{1}\right)+x_{1}-\eta},
$$

so that $h_{1} \geq 0$ since the theorem condition requires the bound $S_{1} \leq 1$. Note that to get (3.6) we use the function

$$
\omega_{1}(x)=v(x) \frac{\beta(x-1)+\eta-x}{\eta-1}
$$

and corresponding equalities $\omega_{1}(1)=\omega_{1}(\eta), \omega_{1}^{\prime}\left(x_{1}\right)=0$.

In summary, we revealed that on some interval $\left[x_{0}, x_{1}\right]$ the function $v(x)$ satisfies the boundary value problem (BVP)

$$
L v(x)=-f_{1}(x), x_{0}<x<x_{1}, \quad \delta_{0} v^{\prime}\left(x_{0}\right)-h_{0} v\left(x_{0}\right)=0, \quad \delta_{1} v^{\prime}\left(x_{1}\right)+h_{1} v\left(x_{1}\right)=0,
$$

where $\delta_{0}=1$ if $\alpha>0$ and $\delta_{1}=1$ if $\beta>0$, in addition, $\delta_{0}=0, h_{0}=1$ if $\alpha<0$, and $\delta_{1}=0, h_{1}=1$ if $\beta<0$. Hence, in view of the variable replacement

$$
t=\left(x_{1}-x_{0}\right)^{-1}\left(x-x_{0}\right),
$$

we get that the function $\tilde{v}(t)=v(x(t))$ satisfies the BVP

$$
\left\{\begin{array}{l}
\tilde{L} \tilde{v}(t) \equiv \varepsilon \tilde{v}^{\prime \prime}(t)+\tilde{a}(t) \tilde{v}^{\prime}(t)-\tilde{b}(t) \tilde{v}(t)=-\tilde{f}_{1}(t), \quad 0<t<1, \\
\tilde{h}_{0} \tilde{v}(0)-\delta_{0} \tilde{v}^{\prime}(0)=0, \quad \tilde{h}_{1} \tilde{v}(1)+\delta_{1} \tilde{v}^{\prime}(1)=0,
\end{array}\right.
$$

where

$$
\tilde{a}(t)=\left(x_{1}-x_{0}\right) a(x(t)), \quad \tilde{b}(t)=\left(x_{1}-x_{0}\right)^{2} b(x(t)), \quad \tilde{f}_{1}(t)=\left(x_{1}-x_{0}\right)^{2} f_{1}(x(t)),
$$




$$
x(t)=\left(x_{1}-x_{0}\right) t+x_{0}, \quad \tilde{a}(t) \geq\left(\eta_{1}-\zeta_{m_{0}}\right) a_{0}, \quad \tilde{b}(t) \geq\left(\eta_{1}-\zeta_{m_{0}}\right)^{2} b_{0}, \quad 0 \leq t \leq 1,
$$

in addition, $\tilde{h}_{0}, \delta_{0}, \tilde{h}_{1}, \delta_{1}$ are defined by the specification

$$
\begin{array}{ll}
\delta_{0}=0, & \tilde{h}_{0}=1 \text { for } \alpha<0, \\
\delta_{0}=1, & \tilde{h}_{0}=\left(x_{1}-x_{0}\right) h_{0} \text { for } 0<\alpha \leq 1, \\
\delta_{1}=0, & \tilde{h}_{1}=1 \text { for } \beta<0, \\
\delta_{1}=1, & \tilde{h}_{1}=\left(x_{1}-x_{0}\right) h_{1} \quad \text { for } 0<\beta \leq 1,
\end{array}
$$

herewith, $\tilde{h}_{1}+\left(\eta_{1}-\zeta_{m_{0}}\right)^{2} b_{0}>0$ since the theorem condition requires $S_{1}<1$ for $b_{0}=0$ (it means that $0<\beta<1$ for $b_{0}=0$ ) and, therefore, we have $\tilde{h}_{1}>0$. Further, for classical solution of BVP (3.9), by virtue of [12, p. 100-103], we get a uniform on $\varepsilon$ stability estimate

$$
|\tilde{v}(t)| \leq C_{4} \max _{0 \leq y \leq 1}|\tilde{L} \tilde{v}(y)|, \quad 0 \leq t \leq 1,
$$

therefore, in view of the variable replacement,

$$
|v(x)| \leq C_{4} \max _{x_{0} \leq y \leq x_{1}}\left|f_{1}(y)\right|, \quad x_{0} \leq x \leq x_{1},
$$

where $C_{4}$ is an $\varepsilon$-independent constant. Since $\zeta \in\left(x_{0}, x_{1}\right)$ and $\eta \in\left(x_{0}, x_{1}\right)$, then

$$
|v(\zeta)| \leq C_{4} \max _{x_{0} \leq x \leq x_{1}}\left|f_{1}(x)\right|, \quad|v(\eta)| \leq C_{4} \max _{x_{0} \leq x \leq x_{1}}\left|f_{1}(x)\right| .
$$

Hence, in view of NLBVC (2.9),

$$
|v(0)| \leq C_{5} \max _{0 \leq x \leq 1}\left|f_{1}(x)\right|, \quad|v(1)| \leq C_{5} \max _{0 \leq x \leq 1}\left|f_{1}(x)\right|,
$$

where $C_{5}=C_{4} \max \{|\alpha|,|\beta|\}$. Now, in view of (3.12), we interpret the solution of NLBVP (2.9) as classical solution of Dirichlet problem

$$
L v(x)=-f_{1}(x), 0<x<1, \quad v(0)=\gamma_{0}, \quad v(1)=\gamma_{1},
$$

where

$$
\left|\gamma_{i}\right| \leq C_{5} \max _{0 \leq x \leq 1}\left|f_{1}(x)\right|, \quad i=0,1 .
$$

Then, by virtue of [12, p. 100-103], we obtain a unifromly stable estimate

$$
|v(x)| \leq C_{6}\left(\left|\gamma_{0}\right|+\left|\gamma_{1}\right|+\max _{0 \leq y \leq 1}|L v(y)|\right), \quad 0 \leq x \leq 1,
$$

where $C_{6}$ is some $\varepsilon$-independent constant. In view of (3.14), the estimate (3.2) is true. Therefore, a uniform on $\varepsilon$ stability estimate (3.1) is proved.

Subcase 2. Put that each one of two conditions (2.2) encloses different sign coefficients. Then $u(x)$ satisfies some reduced condition (2.4). We will prove the estimate (3.2). Further, we admit that $v(0) \neq 0$ and $v(1) \neq 0$, since for the case if $v(0)=0$ or $v(1)=0$ the estimate (3.2) is provable by the same approach which we use here.

Firstly, assume that $\zeta^{-}<\zeta^{+}$in respect of (2.4).

a) If $\operatorname{sign}\left[v(0) v\left(\zeta^{-}\right)\right] \neq 1$ or $\operatorname{sign}\left[v(0) v\left(\zeta^{+}\right)\right] \neq 1$, then there is some point $x_{0}$, $x_{0} \in\left(0, \zeta^{-}\right)$or $x_{0} \in\left(0, \zeta^{+}\right)$correspondingly, so that $v\left(x_{0}\right)=0$.

b) If $\operatorname{sign}\left[v(0) v\left(\zeta^{-}\right)\right]=1$ and $\operatorname{sign}\left[v(0) v\left(\zeta^{+}\right)\right]=1$, then, by virtue of $\mathrm{MV}$ propety [3, p. 1198-1199] in respect of the first nonlocal condition (2.4), we have

$$
\left(1+\left|\alpha^{-}\right|\right) v\left(\zeta_{0}\right)=\alpha^{+} v\left(\zeta^{+}\right)
$$

for some $\zeta_{0} \in\left[0, \zeta^{-}\right]$, herewith $\zeta_{0}<\zeta^{+}$. Then, in view of the condition $S_{0} \leq 1$,

$$
v\left(\zeta_{0}\right)=\alpha_{0} v\left(\zeta^{+}\right), \quad \alpha_{0}=\frac{\alpha^{+}}{1+\left|\alpha^{-}\right|}, \quad 0<\alpha_{0} \leq 1 .
$$


Hence, by using the function

$$
\hat{w}_{0}(x)=v(x) \frac{\left(\alpha_{0}-1\right) x+\zeta^{+}-\alpha_{0} \zeta_{0}}{\zeta^{+}-\zeta_{0}},
$$

we get $\hat{w}_{0}\left(\zeta_{0}\right)=\hat{w}_{0}\left(\zeta^{+}\right)$, so that, by virtue of Rolle's theorem, $\hat{w}^{\prime}\left(x_{0}\right)=0$ at some point $x_{0} \in\left(\zeta_{0}, \zeta^{+}\right)$, and, therefore,

$$
v^{\prime}\left(x_{0}\right)-h_{0} v\left(x_{0}\right)=0, \quad h_{0}=\frac{1-\alpha_{0}}{\left(\alpha_{0}-1\right) x_{0}+\zeta^{+}-\alpha_{0} \zeta_{0}}, \quad h_{0} \geq 0 .
$$

Now, assume that $\zeta^{+}<\zeta^{-}$in respect of (2.4).

a) If $\operatorname{sign}\left[v(0) v\left(\zeta^{+}\right)\right] \neq 1$ or $\operatorname{sign}\left[v(0) v\left(\zeta^{-}\right)\right] \neq 1$, then there is some point $x_{0}$, $x_{0} \in\left(0, \zeta^{+}\right)$or $x_{0} \in\left(0, \zeta^{-}\right)$correspondingly, so that $v\left(x_{0}\right)=0$.

b) If $\operatorname{sign}\left[v(0) v\left(\zeta^{+}\right)\right]=1$ and $\operatorname{sign}\left[v(0) v\left(\zeta^{-}\right)\right]=1$, then there is some value $\tilde{\alpha}_{0}$, $0<\tilde{\alpha}_{0}<\alpha^{+}$, so that, in view of the condition $S_{0} \leq 1$,

$$
v(0)=\tilde{\alpha}_{0} v\left(\zeta^{+}\right), \quad 0<\tilde{\alpha}_{0}<1 .
$$

Hence, by using

$$
\tilde{\omega}_{0}(x)=v(x) \frac{\left(\tilde{\alpha}_{0}-1\right) x+\zeta^{+}}{\zeta^{+}},
$$

we get $\tilde{\omega}_{0}(0)=\tilde{\omega}_{0}\left(\zeta^{+}\right)$, then $\tilde{\omega}^{\prime}\left(x_{0}\right)=0$ at some point $x_{0}, x_{0} \in\left(0, \zeta^{+}\right)$, and, therefore,

$$
v^{\prime}\left(x_{0}\right)-h_{0} v\left(x_{0}\right)=0, \quad h_{0}=\frac{1-\tilde{\alpha}_{0}}{\zeta^{+}-x_{0}\left(1-\tilde{\alpha}_{0}\right)}, \quad h_{0}>0 .
$$

In summary, we revealed that at some point $x_{0}$ the solution of (2.9) satisfies one of the left-side BVC:

$$
\begin{aligned}
\ell_{x_{0}, 1}(v) & \equiv v\left(x_{0}\right)=0,0<x_{0}<\zeta^{-}<\zeta^{+}, \\
\ell_{x_{0}, 2}(v) & \equiv v\left(x_{0}\right)=0,0<x_{0}<\zeta^{+}, 0<\zeta^{-}<\zeta^{+}, \\
\ell_{x_{0}, 3}(v) & \equiv v^{\prime}\left(x_{0}\right)-h_{0} v\left(x_{0}\right)=0, \quad h_{0} \geq 0, \quad 0<x_{0}<\zeta^{+}, 0<\zeta^{-}<\zeta^{+}, \\
\ell_{x_{0}, 4}(v) & \equiv v\left(x_{0}\right)=0,0<x_{0}<\zeta^{+}<\zeta^{-}, \\
\ell_{x_{0}, 5}(v) & \equiv v\left(x_{0}\right)=0,0<x_{0}<\zeta^{-}, 0<\zeta^{+}<\zeta^{-} \\
\ell_{x_{0}, 6}(v) & \equiv v^{\prime}\left(x_{0}\right)-h_{0} v\left(x_{0}\right)=0, \quad h_{0}>0,0<x_{0}<\zeta^{+}<\zeta^{-} .
\end{aligned}
$$

By similar way, we reveal that at some point $x_{1}$ the solution of (2.9) satisfies one of the right-side BVC:

$$
\begin{aligned}
& \ell_{x_{1}, 1}(v) \equiv v\left(x_{1}\right)=0, \quad \eta^{+}<\eta^{-}<x_{1}<1, \\
& \ell_{x_{1}, 2}(v) \equiv v\left(x_{1}\right)=0, \quad \eta^{+}<x_{1}<1, \eta^{+}<\eta^{-}<1, \\
& \ell_{x_{1}, 3}(v) \equiv v^{\prime}\left(x_{1}\right)+h_{1} v\left(x_{1}\right)=0, \quad h_{1} \geq 0, \quad \eta^{+}<x_{1}<1, \quad \eta^{+}<\eta^{-}<1, \\
& \ell_{x_{1}, 4}(v) \equiv v\left(x_{1}\right)=0, \quad \eta^{-}<\eta^{+}<x_{1}<1, \\
& \ell_{x_{1}, 5}(v) \equiv v\left(x_{1}\right)=0, \quad \eta^{-}<x_{1}<1, \eta^{-}<\eta^{+}<1, \\
& \ell_{x_{1}, 6}(v) \equiv v^{\prime}\left(x_{1}\right)+h_{1} v\left(x_{1}\right)=0, \quad h_{1}>0, \quad \eta^{-}<\eta^{+}<x_{1}<1,
\end{aligned}
$$

where similarly (3.16)-(3.17), by using

$$
\hat{w}_{1}(x)=v(x) \frac{\left(\beta_{0}-1\right) x+\eta^{+}-\beta_{0} \eta_{0}}{\eta^{+}-\eta_{0}},
$$

we define

$$
h_{1}=\frac{1-\beta_{0}}{\beta_{0}\left(\eta_{0}-x_{1}\right)+x_{1}-\eta^{+}}, \quad \beta_{0}=\frac{\beta^{+}}{1+\mid \beta^{-\mid}},
$$


for $\ell_{x_{1}, 3}(v)=0$, as well as, similarly (3.18)-(3.19), by using

$$
\tilde{\omega}_{1}(x)=v(x) \frac{\left(\tilde{\beta}_{0}-1\right) x+\eta^{+}-\tilde{\beta}_{0}}{\eta^{+}-1}
$$

for the case if $\operatorname{sign}\left[v(1) v\left(\eta^{+}\right)\right]=\operatorname{sign}\left[v(1) v\left(\eta^{-}\right)\right]=1$, we define

$$
h_{1}=\frac{1-\tilde{\beta}_{0}}{\tilde{\beta}_{0}\left(1-x_{1}\right)+x_{1}-\eta^{+}}
$$

for $\ell_{x_{1}, 6}(v)=0$. Here, $\tilde{\beta}_{0}$ is an appropriate value, so that $v(1)=\tilde{\beta}_{0} v\left(\eta^{+}\right)$, herewith $0<\tilde{\beta}_{0}<\beta^{+}$, so $0<\tilde{\beta}_{0}<1$ since $S_{1} \leq 1$ in view of theorem condition.

Further, let $v(x)$ satisfies some pair of $\mathrm{BVC} \quad \ell_{x_{0}, i}(v)=0, \quad \ell_{x_{1}, j}(v)=0$, $i=1, \ldots, 6, j=1, \ldots, 6$.

2.1. Assume, that $\zeta^{-}, \zeta^{+}, \eta^{-}, \eta^{+} \in\left(x_{0}, x_{1}\right)$. Note, it is always fulfiled for any pair $\ell_{x_{0}, i}(v)=0, \quad \ell_{x_{1}, j}(v)=0, \quad i=1,4,6, \quad j=1,4,6$. Similarly to the Subcase 1 , by virtue of (3.8), we obtain the BVP (3.9)

$$
\tilde{L} \tilde{v}(t)=-\tilde{f}_{1}(t), \quad 0<t<1, \quad \tilde{h}_{0} \tilde{v}(0)-\delta_{0} \tilde{v}^{\prime}(0)=0, \quad \tilde{h}_{1} \tilde{v}(1)+\delta_{1} \tilde{v}^{\prime}(1)=0,
$$

where

$$
\begin{aligned}
& \delta_{0}=0, \tilde{h}_{0}=1 \quad \text { for conditions } \ell_{x_{0}, i}(v)=0, i=1,2,4,5 \\
& \delta_{0}=1, \tilde{h}_{0}=\left(x_{1}-x_{0}\right) h_{0} \text { for conditions } \ell_{x_{0}, i}(v)=0, i=3,6, \\
& \delta_{1}=0, \tilde{h}_{1}=1 \text { for conditions } \ell_{x_{1}, j}(v)=0, j=1,2,4,5 \\
& \delta_{1}=1, \tilde{h}_{1}=\left(x_{1}-x_{0}\right) h_{1} \text { for conditions } \ell_{x_{1}, j}(v)=0, j=3,6,
\end{aligned}
$$

herewith, $\tilde{h}_{1}+\left(\eta_{1}-\zeta_{m_{0}}\right)^{2} b_{0}>0$ for $\ell_{x_{1}, j}(v)=0, j=1,2,4,5,6$ since $\tilde{h}_{1}>0$, in addition, $\tilde{h}_{1}+\left(\eta_{1}-\zeta_{m_{0}}\right)^{2} b_{0}>0$ for $\ell_{x_{1}, 3}(v)=0$ in view of the theorem requirement $S_{1}<1$. Then the estimate (3.10) holds for $\tilde{v}(x)$. Since (3.10) results in (3.11), then, in view of $\zeta^{-}, \zeta^{+}, \eta^{-}, \eta^{+} \in\left(x_{0}, x_{1}\right)$, we get

$$
\begin{array}{ll}
\left|v\left(\zeta^{-}\right)\right| \leq C_{4} \max _{x_{0} \leq x \leq x_{1}}\left|f_{1}(x)\right|, \quad & \left|v\left(\zeta^{+}\right)\right| \leq C_{4} \max _{x_{0} \leq x \leq x_{1}}\left|f_{1}(x)\right|, \\
\left|v\left(\eta^{-}\right)\right| \leq C_{4} \max _{x_{0} \leq x \leq x_{1}}\left|f_{1}(x)\right|, & \left|v\left(\eta^{+}\right)\right| \leq C_{4} \max _{x_{0} \leq x \leq x_{1}}\left|f_{1}(x)\right| .
\end{array}
$$

In view of (3.20) and (3.21), the estimate (3.12) follows from NLBVC (2.9) for $C_{5}=C_{4} \max \left\{\left|\alpha^{-}\right|+\left|\alpha^{+}\right|,\left|\beta^{-}\right|+\left|\beta^{+}\right|\right\}$. Therefore, we can interpret the solution of NLBVP (2.9) as classical solution of the Dirichlet's problem (3.13) and, by virtue of [12, p. 100-103], state that a unifromly stable estimate (3.15) holds. Hence, in view of (3.14), we get the estimate (3.11). At least, the validity of (3.11) is sufficient to confirm that (3.1) is true.

2.2. Assume, that only one of two points $\zeta^{-}$or $\zeta^{+}$belongs to $\left(x_{0}, x_{1}\right)$, as well as, only one of two points $\eta^{-}$or $\eta^{+}$belongs to $\left(x_{0}, x_{1}\right)$ (it is available in respect of any pair of the conditions $\left.\ell_{x_{0}, i}(v)=0, \quad \ell_{x_{1}, j}(v)=0, \quad i=2,3,5, \quad j=2,3,5\right)$. Then, since (3.10)-(3.11) holds, then one of two estimates (3.20) holds, as well as, one of two estimates (3.21) holds too. Thus, the NLBVP (2.9) is reduciable to the problem

$$
L v(x)=-f_{1}(x), 0<x<1, v(0)=\alpha^{*} v\left(\zeta^{*}\right)+\varphi_{0}^{*}, v(1)=\beta^{*} v\left(\eta^{*}\right)+\varphi_{1}^{*},
$$

where

$$
\left|\varphi_{0}^{*}\right| \leq C_{5} \max _{0 \leq x \leq 1}\left|f_{1}(x)\right|, \quad\left|\varphi_{1}^{*}\right| \leq C_{5} \max _{0 \leq x \leq 1}\left|f_{1}(x)\right|
$$


$C_{5}=C_{4} \max \left\{\left|\alpha^{-}\right|+\left|\alpha^{+}\right|,\left|\beta^{-}\right|+\left|\beta^{+}\right|\right\}$, the pair $\alpha^{*}$ and $\zeta^{*}$ is performed by $\alpha^{-}$ and $\zeta^{-}$, or by $\alpha^{+}$and $\zeta^{+}$correspondingly, the pair $\beta^{*}$ and $\eta^{*}$ is performed by $\beta^{+}$ and $\eta^{+}$, or by $\beta^{-}$and $\eta^{-}$. So, in view of the theorem condition, $-\infty<\alpha^{*} \leq 1$, $-\infty<\beta^{*} \leq 1, \beta^{*}<1$ if $b_{0}=0$. Now, firstly by virtue of Lemma 2.1 in respect of the problem (3.22), and then, by reasoning similar Section 1, we obtain the analogy of (3.1) for the problem (3.22), i.e.,

$$
|v(x)| \leq C\left(\left|\varphi_{0}^{*}\right|+\left|\varphi_{1}^{*}\right|+\max _{0 \leq y \leq 1}\left|f_{1}(y)\right|\right), \quad 0 \leq x \leq 1 .
$$

Then, in view of (3.23), the estimate (3.24) results in (3.2), and, therefore, the estimate (3.1) is true.

2.3. Assume, that three of four points $\zeta^{-}, \zeta^{+}, \eta^{-}, \eta^{+}$belong to $\left(x_{0}, x_{1}\right)$. Then, by combined reasoning of 2.1-2.2, one can prove that the estimate (3.1) is true.

Subcase 3. Assume, that one of two conditions (2.2) encloses the coefficients of the same sign, but another one encloses different sign coefficients. Then (3.1) can be proved by virtue of combined approach of Subcases 1-2. Theorem 3.1 is proved.

\section{The existence And uniqueness}

Firstly, we prove

Lemma 4.1. Let (2.3) and the condition $\boldsymbol{A}$ are fulfiled, $S_{i} \leq 1, \varphi_{i} \neq 0, i=0,1$. If $u(x)$ is some classical solution of NLBVP (2.1),(2.2), then

$$
v(x)=u(x)+\varphi_{0} q_{0}(x)+\varphi_{1} q_{1}(x)
$$

is classical solution of the problem

$$
L v(x)=-f_{1}(x), 0<x<1, \quad \ell_{0}(v)=0, \quad \ell_{1}(v)=0
$$

for $f_{1}(x)=f(x)-\varphi_{0} L q_{0}(x)-\varphi_{1} L q_{1}(x)$, where $q_{0}(x)=c_{0}(1-x) \prod_{l=1}^{m_{1}}\left(\eta_{l}-x\right)$, $q_{1}(x)=c_{1} x \prod_{k=1}^{m_{0}}\left(x-\zeta_{k}\right)$, herewith an appropriate constant $c_{i} \in \boldsymbol{R}, \quad c_{i} \neq 0, i=0,1$.

If $v(x)$ is some classical solution of (4.1), then $u(x)=v(x)-\varphi_{0} q_{0}(x)-\varphi_{1} q_{1}(x)$ is classical solution of $N L B V P$ (2.1), (2.2) for $f(x)=f_{1}(x)+\varphi_{0} L q_{0}(x)+\varphi_{1} L q_{1}(x)$.

Let only one of $\varphi_{i}, i \in\{0,1\}$ be nonzero, put $\varphi_{i_{*}} \neq 0, i_{*} \in\{0,1\}$. If $u(x)$ is some classical solution of NLBVP (2.1),(2.2), then $v(x)=u(x)+\varphi_{i_{*}} q_{i_{*}}(x)$ is classical solution of the problem (4.1) for the function $f_{1}(x)=f(x)-\varphi_{i_{*}} L q_{i_{*}}(x)$. Vice versa, if $v(x)$ is some classical solution of (4.1), then $u(x)=v(x)-\varphi_{i_{*}} q_{*}(x)$ is classical solution of NLBVP (2.1),(2.2) for $f(x)=f_{1}(x)+\varphi_{i_{*}} L q_{i_{*}}(x)$.

Proof. Put $\varphi_{i} \neq 0, i=0,1$. Obviously that $L v(x)=-f_{1}(x), \quad 0<x<1$ for $q_{i}(x)$ and any nonzero $c_{i}, i=0,1$. Let us find $c_{i}, i=0,1$, so that the $v(x)$ will satisfy NLBVC (4.1). Note,

$$
\ell_{0}\left(q_{1}\right)=0, \ell_{1}\left(q_{0}\right)=0 .
$$

Since $v(x)$ has to satisfy the NLBVC (4.1), then the expressions

$$
l_{0}(v)=\varphi_{0}\left[1+\ell_{0}\left(q_{0}\right)\right]=0, \quad l_{1}(v)=\varphi_{1}\left[1+\ell_{1}\left(q_{1}\right)\right]=0
$$

have to be true, therefore,

$$
1+\ell_{0}\left(q_{0}\right)=0, \quad 1+\ell_{1}\left(q_{1}\right)=0
$$


have to be true too. Hence,

$$
c_{0}=-\left(E_{0}\right)^{-1}, \quad c_{1}=-\left(D_{0}\right)^{-1},
$$

where

$$
\begin{aligned}
& E_{0}=\prod_{l=1}^{m_{1}} \eta_{l}-\sum_{k=1}^{m_{0}} \alpha_{k}\left(1-\zeta_{k}\right) \prod_{l=1}^{m_{1}}\left(\eta_{l}-\zeta_{k}\right), \\
& D_{0}=\prod_{k=1}^{m_{0}}\left(1-\zeta_{k}\right)-\sum_{l=1}^{m_{1}} \beta_{l} \eta_{l} \prod_{k=1}^{m_{0}}\left(\eta_{l}-\zeta_{k}\right),
\end{aligned}
$$

herewith $E_{0} \neq 0, D_{0} \neq 0$, moreover, $E_{0}>0, D_{0}>0$. Actually, by virtue of the condition $S_{0} \leq 1$ in respect of (4.6), we have

$$
E_{0}>\left\{\begin{array}{l}
\prod_{l=1}^{m_{1}} \eta_{l}>0, \text { if } \alpha_{k}<0, k=1, \ldots, m_{0}, \\
(1-\alpha) \prod_{l=1}^{m_{1}} \eta_{l} \geq 0, \text { if } \alpha_{k}>0, k=1, \ldots, m_{0}, \\
\left(1-S_{0}\right) \prod_{l=1}^{m_{1}} \eta_{l} \geq 0, \text { if all } \alpha_{k}, k=1, \ldots, m_{0} \text { have not the same sign, }
\end{array}\right.
$$

where, in view of (2.6), $S_{0}=\alpha^{+}$for $\zeta^{+}<\zeta^{-}, S_{0}=\alpha^{-}+\alpha^{+}$for $\zeta^{-}<\zeta^{+}$. Indeed, it is clear for the case if all coefficients $\alpha_{k}, k=1, \ldots, m_{0}$ have the same sign, as well as, for the case if $\zeta^{+}<\zeta^{-}$. Let us confirm that $E_{0}>0$ for the case if all $\alpha_{k}, k=1, \ldots, m_{0}$ have not the same sign and $\zeta^{-}<\zeta^{+}$. In view of (4.6),

$$
E_{0}>\prod_{l=1}^{m_{1}} \eta_{l}-\left(1-\zeta_{m_{\iota}}\right) \alpha^{-} \prod_{l=1}^{m_{1}}\left(\eta_{l}-\zeta_{m_{\iota}}\right)-\left(1-\zeta_{m_{\iota}+1}\right) \alpha^{+} \prod_{l=1}^{m_{1}}\left(\eta_{l}-\zeta_{m_{\iota}+1}\right),
$$

then

$$
E_{0}>\prod_{l=1}^{m_{1}} \eta_{l}-\left(1-\zeta_{m_{\iota}}\right)\left(\alpha^{-}+\alpha^{+}\right) \prod_{l=1}^{m_{1}}\left(\eta_{l}-\zeta_{m_{\iota}}\right)
$$

Hence, $E_{0}>\prod_{l=1}^{m_{1}} \eta_{l}>0$ for $-\infty<S_{0} \leq 0, E_{0}>\left[1-\left(\alpha^{-}+\alpha^{+}\right)\right] \prod_{l=1}^{m_{1}} \eta_{l} \geq 0$ for $0<S_{0} \leq 1$, where $S_{0}=\alpha^{-}+\alpha^{+}$. Thus, we proved finally that $E_{0}>0$, then $E_{0} \neq 0$, therefore, the constant $c_{0}$ is definable by the first formula (4.5). Similarly, by virtue of the condition $S_{1} \leq 1$ for (4.7), it is easy to confirm that $D_{0}>0$ and prove that the constant $c_{1}$ is definable by the second formula of (4.5).

Let us prove second statement of lemma. Obviously,

$$
L u(x)=L v(x)-\sum_{i=1}^{2} \varphi_{i} L q_{i}(x)=-f_{1}(x)-\sum_{i=1}^{2} \varphi_{i} L q_{i}(x)=-f(x), \quad 0<x<1,
$$

in addition, since $v(x)$ satisfies NLBVC (4.1), then, in view of (4.2)-(4.5), we get

$$
\begin{aligned}
& \ell_{0}(u)=\ell_{0}(v)-\varphi_{0} \ell_{0}\left(q_{0}\right)-\varphi_{1} \ell_{0}\left(q_{1}\right)=-\varphi_{0} \ell_{0}\left(q_{0}\right)=\varphi_{0}, \\
& \ell_{1}(u)=\ell_{1}(v)-\varphi_{0} \ell_{1}\left(q_{0}\right)-\varphi_{1} \ell_{1}\left(q_{1}\right)=-\varphi_{1} \ell_{1}\left(q_{1}\right)=\varphi_{1} .
\end{aligned}
$$

To finish this proof, by the same way as in above it is easy to confirm, that the third statement of lemma is true. Lemma 4.1 is proved.

Theorem 4.2. Let $a(x) \geq a_{0}>0, b(x) \geq b_{0} \geq 0$ for $x \in[0,1]$. Let (2.3) and the condition $\boldsymbol{A}$ are fulfilled. If $S_{i} \leq 1, i=0,1$ and, in addition, $S_{1}<1$ if $b_{0}=0$, then classical solution of NLBVP (2.1),(2.2) exists and is a unique. 
Proof. Because all conditions of Theorem 3.1 are fulfiled, then the uniqueness of classical solution follows from stabilty estimate (3.1).

In view of Lemma 4.1, to prove the existence, it is sufficient to establish that classical solution of the differential problem (4.1) exists. The problem (4.1) is equivalent to the differential problem

$$
\left[k(x) v^{\prime}\right]^{\prime}-q(x) v=-\tilde{f}_{1}(x), \quad 0<x<1, \quad \ell_{0}(v)=0, \quad \ell_{1}(v)=0,
$$

where

$$
k(x)=\exp \left(\frac{1}{\varepsilon} \int_{0}^{x} a(t) d t\right), q(x)=b(x) k(x), \quad \tilde{f}_{1}(x)=f_{1}(x) k(x),
$$

therefore, it will be sufficient to prove that classical solution of (4.8) exists. To prove it let us use the fact that for any continuous function $F(x), x \in[0,1]$ the differential problem

$$
\left[k(x) v^{\prime}\right]^{\prime}-v / k(x)=F(x), \quad 0<x<1, \quad \ell_{0} v=0, \quad \ell_{1} v=0
$$

has the solution

$$
v(x)=A \sinh (P(x))+B \cosh (P(x))+\int_{0}^{x} \sinh (P(x)-P(t)) F(t) d t,
$$

where

$$
\begin{gathered}
P(x)=\int_{0}^{x}(k(\tau))^{-1} d \tau, \\
A=-\left[\ell_{1}\left(s_{P}\right)\right]^{-1}\left\{B \ell_{1}\left(c_{P}\right)+\ell_{1}\left(\int_{0}^{x} \sinh (P(x)-P(t)) F(t) d t\right)\right\}, \\
B=-\left[\ell_{0}\left(c_{P}\right)-\ell_{0}\left(s_{P}\right) \frac{\ell_{1}\left(c_{P}\right)}{\ell_{1}\left(s_{P}\right)}\right]^{-1}\left\{\ell_{0}\left(\int_{0}^{x} \sinh (P(x)-P(t)) F(t) d t\right)\right. \\
\left.-\frac{\ell_{0}\left(s_{P}\right)}{\ell_{1}\left(s_{P}\right)} \ell_{1}\left(\int_{0}^{x} \sinh (P(x)-P(t)) F(t) d t\right)\right\},
\end{gathered}
$$

herewith for the convenience we use next designation

$$
s_{P}(x)=\sinh (P(x)), \quad c_{P}(x)=\cosh (P(x)) .
$$

Actually, written in the square brackets expression is nonzero for $A$ and $B$. Indeed, since the function $P(x)$ is nonnegative and strictly increasing in $[0,1]$, then

$$
\ell_{1}\left(s_{P}\right)>\left\{\begin{array}{l}
s_{P}(1)>0, \quad \text { if } \beta_{l}<0, \quad l=1, \ldots, m_{1}, \\
s_{P}(1)-\beta s_{P}\left(\eta_{m_{1}}\right)>0, \quad \text { if } \beta_{l}>0, \quad l=1, \ldots, m_{1} .
\end{array}\right.
$$

If all coefficients $\beta_{l}, l=1, \ldots, m_{1}$ have not the same sign, then, in view of the MV property [3, p. 1198-1199],

$$
\ell_{1}\left(s_{P}\right)=s_{P}(1)-\beta^{+} s_{P}\left(\tilde{\eta}^{+}\right)-\beta^{-} s_{P}\left(\tilde{\eta}^{-}\right),
$$


where $\left.\tilde{\eta}^{+}, \tilde{\eta}^{-} \in\left[\eta_{1}, \eta_{m_{1}}\right]\right]^{3}$ In view of (4.14), if $\tilde{\eta}^{-}<\tilde{\eta}^{+}$, then

$$
\ell_{1}\left(s_{P}\right)>s_{P}(1)-\beta^{+} s_{P}\left(\eta_{m_{1}}\right)>0 \text {. }
$$

If $\tilde{\eta}^{+}<\tilde{\eta}^{-}$, then $\beta^{+}+\beta^{-}=S_{1}$, then, in view of (4.14), we get

$$
\ell_{1}\left(s_{P}\right)>s_{P}(1)-S_{1} s_{P}\left(\eta_{m_{\kappa}}\right)>0 \text {. }
$$

In summary, we proved that

$$
\ell_{1}\left(s_{P}\right)>0 .
$$

Now, let us prove that the expression inside square brackets for $B$ is nonzero too.

Let us denote this expression by $G$, i.e.,

$$
G=\ell_{0}\left(c_{P}\right)-\ell_{0}\left(s_{P}\right) \times \frac{\ell_{1}\left(c_{P}\right)}{\ell_{1}\left(s_{P}\right)} .
$$

Additionally, we denote

$$
\Phi(x)=c_{P}(x)-s_{P}(x) \times \frac{\ell_{1}\left(c_{P}\right)}{\ell_{1}\left(s_{P}\right)} .
$$

Since

$$
G=1-\sum_{k=1}^{m_{0}} \alpha_{k} c_{P}\left(\zeta_{k}\right)+\sum_{k=1}^{m_{0}} \alpha_{k} s_{P}\left(\zeta_{k}\right) \times \frac{\ell_{1}\left(c_{P}\right)}{\ell_{1}\left(s_{P}\right)},
$$

then, by virtue of MV property, there are some points $\rrbracket^{4} \tilde{\zeta}, \tilde{\zeta}^{+}, \tilde{\zeta}^{-} \in\left[\zeta_{1}, \zeta_{m_{0}}\right]$, so that

$$
G=\left\{\begin{array}{l}
1-\alpha \Phi(\tilde{\zeta}), \quad \text { if } \quad \alpha_{k}, \quad k=1, \ldots, m_{0} \text { have the same sign, } \\
1-\alpha^{+} \Phi\left(\tilde{\zeta}^{+}\right)-\alpha^{-} \Phi\left(\tilde{\zeta}^{-}\right), \text {if all } \alpha_{k}, k=1, \ldots, m_{0} \text { have not } \\
\text { the same sign }
\end{array}\right.
$$

Further, in respect of $\Phi(x), x \in\left[0, \zeta_{m_{0}}\right]$, we have

$$
\begin{gathered}
\Phi(x)=c_{P}(x)-s_{P}(x) \times \frac{c_{P}(1)-\sum_{l=1}^{m_{1}} \beta_{l} c_{P}\left(\eta_{l}\right)}{s_{P}(1)-\sum_{l=1}^{m_{1}} \beta_{l} s_{P}\left(\eta_{l}\right)} \\
=\left[\sinh (P(1)-P(x))-\sum_{l=1}^{m_{1}} \beta_{l} \sinh \left(P\left(\eta_{l}\right)-P(x)\right)\right] \times \frac{1}{\ell_{1}\left(s_{P}\right)} .
\end{gathered}
$$

Hence, by virtue of MV property [3, p. 1198-1199], for $x \in\left[0, \zeta_{m_{0}}\right]$ we obtain that

$$
\Phi(x)=\frac{\sinh (P(1)-P(x))-\beta \sinh (P(\hat{\eta})-P(x))}{\ell_{1}\left(s_{P}\right)}
$$

for some $\hat{\eta} \in\left[\eta_{1}, \eta_{m_{1}}\right]$ if $\beta_{l}, l=1, \ldots, m_{1}$ have the same sign. However, if all $\beta_{l}$, $l=1, \ldots, m_{1}$ have not the same sign 5 , then

$$
\Phi(x)=\frac{\sinh (P(1)-P(x))-\beta^{+} \sinh \left(P\left(\hat{\eta}^{+}\right)-P(x)\right)-\beta^{-} \sinh \left(P\left(\hat{\eta}^{-}\right)-P(x)\right)}{\ell_{1}\left(s_{P}\right)}
$$

\footnotetext{
${ }^{3}$ Note, $\tilde{\eta}^{+}<\tilde{\eta}^{-}$if $\eta^{+}<\eta^{-}$, or, alternatively, $\tilde{\eta}^{-}<\tilde{\eta}^{+}$if $\eta^{-}<\eta^{+}$, where $\eta^{+}$and $\eta^{-}$ are some designated points respectively NLBVC (2.4).

${ }^{4}$ Note, $\tilde{\zeta}^{+}<\tilde{\zeta}^{-}$if $\zeta^{+}<\zeta^{-}$, or, alternatively, $\tilde{\zeta}^{-}<\tilde{\zeta}^{+}$if $\zeta^{-}<\zeta^{+}$, where $\zeta^{+}$and $\zeta^{-}$ are some designated points respectively NLBVC (2.4).

${ }^{5}$ Note, $\hat{\eta}^{+}<\hat{\eta}^{-}$if $\eta^{+}<\eta^{-}$, or, alternatively $\hat{\eta}^{-}<\hat{\eta}^{+}$if $\eta^{-}<\eta^{+}$, where $\eta^{+}$and $\eta^{-}$ are defined in respect of NLBVC (2.4).
} 
for some $\hat{\eta}^{+} \in\left[\eta_{1}, \eta_{m_{1}}\right], \hat{\eta}^{-} \in\left[\eta_{1}, \eta_{m_{1}}\right]$. Hence, in view of (4.15), for $x \in\left[0, \zeta_{m_{0}}\right]$ we have:

$$
\begin{gathered}
\Phi(x)>\frac{\sinh (P(1)-P(x))}{\ell_{1}\left(s_{P}\right)}, \text { if } \beta<0 ; \\
\Phi(x)>\frac{\sinh (P(1)-P(x))-\beta \sinh (P(\hat{\eta})-P(x))}{\ell_{1}\left(s_{P}\right)}, \text { if } 0<\beta \leq 1 ; \\
\ell_{1}\left(s_{P}\right)
\end{gathered}
$$

herewith $S_{1}=\beta^{+}+\beta^{-}$;

$$
\Phi(x)>\frac{\sinh (P(1)-P(x))-S_{1} \sinh \left(P\left(\hat{\eta}^{+}\right)-P(x)\right)}{\ell_{1}\left(s_{P}\right)}, \text { if } \hat{\eta}^{-}<\hat{\eta}^{+},
$$

herewith $S_{1}=\beta^{+}$. Since $S_{1} \leq 1$, then for $x \in\left[0, \zeta_{m_{0}}\right]$, in view of (4.17)-(4.20), we obtain the inequality

$$
\Phi(x)>0 .
$$

Further, for the case if $\beta_{l}, l=1, \ldots, m_{1}$ have the same sign we get

$$
\Phi^{\prime}(x)=-\frac{\sinh (P(1)-P(x))-\beta \sinh (P(\hat{\eta})-P(x))}{k(x) \ell_{1}\left(s_{P}\right)},
$$

for the case if all $\beta_{l}, l=1, \ldots, m_{1}$ are not of the same sign we get

$\Phi^{\prime}(x)=-\frac{\sinh (P(1)-P(x))-\beta^{+} \sinh \left(P\left(\hat{\eta}^{+}\right)-P(x)\right)-\beta^{-} \sinh \left(P\left(\hat{\eta}^{-}\right)-P(x)\right)}{k(x) \ell_{1}\left(s_{P}\right)}$.

Hence,

$$
\Phi^{\prime}(x)=-\frac{\Phi(x)}{k(x)} .
$$

Now, in view of $(4.21)$, for $x \in\left[0, \zeta_{m_{0}}\right]$ we have

$$
\Phi^{\prime}(x)<0 .
$$

Then $\Phi(x)$ is strictly decreasing positive function in $\left[0, \zeta_{m_{0}}\right]$, in addition, in view of $(4.11), \Phi(0)=1$, therefore, $0<\Phi(x)<1$ for $x \in\left(0, \zeta_{m_{0}}\right]$. Hence, in view of (4.16)-(4.20), we get

$$
G>\left\{\begin{array}{l}
1-\left(\alpha^{+}+\alpha^{-}\right) \Phi\left(\tilde{\zeta}^{-}\right), \text {if } \tilde{\zeta}^{-}<\tilde{\zeta}^{+} \\
1-\alpha^{+} \Phi\left(\tilde{\zeta}^{+}\right), \text {if } \tilde{\zeta}^{+}<\tilde{\zeta}^{-}, \\
1-\Phi\left(\zeta_{1}\right), \text { if } \alpha_{k}, k=1, \ldots, m_{0} \text { have the same sign. }
\end{array}\right.
$$

Since $S_{0} \leq 1$, then

$$
G>0 .
$$

Thus, in view of (4.15) and (4.24), the coefficients $A$ and $B$ are uniquely definable in respect of the formula (4.10), i.e., the function $v(x)$ is the solution of the differential problem (4.9). Further, by substituting

$$
F(x)=[q(x)-1 / k(x)] v(x)-\tilde{f}_{1}(x)
$$

into the equation (4.9), we obtain that the problem (4.9) is equivivalent to the Fredholm's integral equation of the second kind

$$
v(x)=\int_{0}^{1} K(x, t) v(t) d t+\hat{f}(x)
$$


where

herewith:

$$
\left\{\begin{aligned}
K(x, t) & =K_{0}(x, t)+\sum_{k=1}^{m_{0}}\left[Z_{k}(x, t)+\tilde{Z}_{k}(x, t)\right] \\
& +\sum_{l=1}^{m_{1}}\left[H_{l}(x, t)+\tilde{H}_{l}(x, t)\right]+\sum_{i=1}^{3} R_{i}(x, t)
\end{aligned}\right.
$$

$$
K_{0}(x, t)=\left\{\begin{array}{l}
\sinh (P(x)-P(t))[q(t)-1 / k(t)], \quad \text { if }(x, t) \in Q_{x}, \\
0, \quad \text { if }(x, t) \in \bar{Q}_{x}
\end{array}\right.
$$

for $Q_{x}=\{0 \leq x \leq 1,0 \leq t \leq x\}, \bar{Q}_{x}=\{0 \leq x \leq 1, x \leq t \leq 1\}$;

$$
Z_{k}(x, t)=\left\{\begin{array}{l}
G^{-1} \alpha_{k} c_{P}(x) \sinh \left(P\left(\zeta_{k}\right)-P(t)\right)[q(t)-1 / k(t)], \quad \text { if }(x, t) \in Q_{\zeta_{k}}, \\
0, \quad \text { if }(x, t) \in \bar{Q}_{\zeta_{k}}
\end{array}\right.
$$

and

$$
\tilde{Z}_{k}(x, t)=-\frac{\ell_{1}\left(c_{P}\right) Z_{k}(x, t)}{\ell_{1}\left(s_{P}\right)} \tanh (P(x))
$$

for $Q_{\zeta_{k}}=\left\{0 \leq x \leq 1,0 \leq t \leq \zeta_{k}\right\}, \bar{Q}_{\zeta_{k}}=\left\{0 \leq x \leq 1, \zeta_{k} \leq t \leq 1\right\}, k=1, \ldots, m_{0}$;

$H_{l}(x, t)=\left\{\begin{array}{l}{\left[\ell_{1}\left(s_{P}\right) G\right]^{-1} \beta_{l} \ell_{0}\left(s_{P}\right) c_{P}(x) \sinh \left(P\left(\eta_{l}\right)-P(t)\right)[q(t)-1 / k(t)], \text { if }(x, t) \in Q_{\eta_{l}},} \\ 0, \quad \text { if }(x, t) \in \bar{Q}_{\eta_{l}}\end{array}\right.$ and

$$
\tilde{H}_{l}(x, t)=-\frac{G H_{l}(x, t)}{\ell_{1}\left(s_{P}\right)} \tanh (P(x))
$$

for $Q_{\eta_{l}}=\left\{0 \leq x \leq 1,0 \leq t \leq \eta_{l}\right\}, \bar{Q}_{\eta_{l}}=\left\{0 \leq x \leq 1, \eta_{l} \leq t \leq 1\right\}, l=1, \ldots, m_{1}$; in addition, for $(x, t) \in Q_{1}, Q_{1}=\{0 \leq x \leq 1,0 \leq t \leq 1\}$

$$
\begin{gathered}
R_{1}(x, t)=\frac{\ell_{0}\left(s_{P}\right) c_{P}(x)}{G \ell_{1}\left(s_{P}\right)} \sinh (P(1)-P(t))[q(t)-1 / k(t)], \\
R_{2}(x, t)=-\frac{\ell_{1}\left(c_{P}\right) R_{1}(x, t)}{\ell_{1}\left(s_{P}\right)} \tanh (P(x))
\end{gathered}
$$

and

$$
R_{3}=-\frac{s_{P}(x)}{\ell_{1}\left(s_{P}\right)} \sinh (P(1)-P(t))[q(t)-1 / k(t)]
$$

at least for $x \in[0,1]$

$$
\left\{\begin{array}{l}
\hat{f}(x)=-G^{-1} c_{P}(x) T_{2}+\left[\ell_{1}\left(s_{P}\right)\right]^{-1} s_{P}(x) T_{1} \\
\quad-\left[G \ell_{1}\left(s_{P}\right)\right]^{-1} \ell_{1}\left(c_{P}\right) s_{P}(x) c_{P}(x) T_{2}-\int_{0}^{x} \sinh (P(x)-P(t)) \tilde{f}_{1}(t) d t,
\end{array}\right.
$$

where

$$
\begin{gathered}
T_{1}=\ell_{1}\left(\int_{0}^{x} \sinh (P(x)-P(t)) \tilde{f}_{1}(t) d t\right), \\
T_{2}=\frac{\ell_{0}\left(s_{P}\right)}{\ell_{1}\left(s_{P}\right)} T_{1}-\ell_{0}\left(\int_{0}^{x} \sinh (P(x)-P(t)) \tilde{f}_{1}(t) d t\right) .
\end{gathered}
$$

Since the summands of $(4.27)$ are continuous functions in $[0,1] \times[0,1]$, then the sum $K(x, t)$ is also the continuous function in $[0,1] \times[0,1]$. Therefore, the Fredholm's alternative holds for the integral equation (4.26) in respect of the Hilbert space $L_{2}(0,1)$. Because $\tilde{f}_{1}(x)=f_{1}(x) k(x), f_{1}(x) \in C[0,1]$, then, in view of the formula 
for $\hat{f}(x)$, we have $\hat{f}(x) \in C[0,1]$. Since $K(x, t) \in C([0,1] \times[0,1]), \hat{f}(x) \in C[0,1]$, then, belonged to $L_{2}(0,1)$ solution of the integral equation (4.26) belongs to $C[0,1]$ actually. Then for $v(x) \in C[0,1], k(x) \in C^{1}([0,1]$ and $q(x) \in C[0,1]$, the integral $\int_{0}^{1} K(x, t) v(t) d t$, as the function of $x$, belongs to $C^{2}[0,1]$. In addition, from the formula for $\hat{f}(x)$ it follows that $\hat{f}(x) \in C^{2}[0,1]$ since $\tilde{f}_{1}(x) \in C[0,1]$. In summary, any solution from $L_{2}(0,1)$ of the integral equation (4.26) belongs to $C^{2}[0,1]$. Then it is sufficient to prove that (4.26) has only the trivial solution if $\hat{f}(x) \equiv 0$ on $[0,1]$.

Put $\hat{f}(x) \equiv 0$ on $[0,1]$ for the integral equation $(4.26)$. Then $\tilde{f}_{1}(x) \equiv 0$ on $[0,1]$. Indeed, since $\hat{f}(0)=0$, then, in view of (4.12) and (4.28), $T_{2}=0$. Therefore,

$$
\hat{f}(x)=\frac{s_{P}(x)}{\ell_{1}\left(s_{P}\right)} T_{1}-\int_{0}^{x} \sinh (P(x)-P(t)) \tilde{f}_{1}(t) d t .
$$

Hence,

$$
\hat{f}^{\prime}(x)=\frac{c_{P}(x)}{k(x) \ell_{1}\left(s_{P}\right)} T_{1}-\frac{1}{k(x)} \int_{0}^{x} \cosh (P(x)-P(t)) \tilde{f}_{1}(t) d t,
$$

herewith, since we put $\hat{f}(x) \equiv 0$, then $\hat{f}^{\prime}(x) \equiv 0$ on $[0,1]$. Since $\hat{f}^{\prime}(0)=0$, then $T_{1}=0$, therefore,

$$
\int_{0}^{x} \cosh (P(x)-P(t)) \tilde{f}_{1}(t) d t \equiv 0
$$

on $[0,1]$. Hence, similarly [13, p. 46], we obtain that $\tilde{f}_{1}(x) \equiv 0$ on $[0,1]$. Since $\tilde{f}_{1}(x)=f_{1}(x) k(x)$, then $f_{1}(x) \equiv 0$ on $[0,1]$. Since $f_{1}(x) \equiv 0$, then, in view of Theorem 3.1, the NLBVP (4.1) has only trivial solution $v(x) \equiv 0$. Hence, because the problem (4.1) is equivalent to the differential problem (4.8), (4.8) is equivalent to the differential problem (4.9) for the defined by (4.25) function $F(x)$ and (4.9) is equivivalent to the integral equation (4.26), then (4.26) has only trivial solution if $\hat{f}(x) \equiv 0$ on $[0,1]$.

Thus we proved that the solution $v(x)$ of the integral equation (4.26) exits and belongs to $C^{2}[0,1]$, then, in view of the equivalence, $v(x)$ is classical solution of NLBVP (4.1) at the same time. By virtue of Lemma 4.1, since classical solution $v(x)$ of NLBVP (4.1) exits, then classical solution $u(x)$ of NLBVP $(2.1),(2.2)$ exists too. Theorem 4.2 is proved.

\section{Ill-Posed StATEMEnt EXAMPles}

Next examples show that stated for NLBVP (2.1)-(2.2) condition on $S_{i}, i=0,1$ is essential for well-posedness of the problem.

Example 1. The problem

$$
\varepsilon u^{\prime \prime}(x)+a(x) u^{\prime}(x)=0,0<x<1, u(0)=u(\zeta), u(1)=u(\eta)
$$

is ill-posed, it has infinite number of solutions $u(x)=$ const. It shows the essentiality of condition $S_{1}<1$ for the case if $b_{0}=0$.

Example 2. The problem

$$
\varepsilon u^{\prime \prime}(x)+a(x) u^{\prime}(x)=0,0<x<1, u(0)=u(\zeta), u(1)=u(\eta)+1
$$


is ill-posed, it has no solution for $0<\zeta<\eta<1$. Indeed, assume that some solution of the problem exists, then $u^{\prime}(\xi)=0$ at some point $\xi \in(0, \zeta)$, therefore, $u^{\prime}(x) \equiv 0$ on $[\xi, 1]$, then $u(x)=$ const on $[0,1]$, so that it conflicts with the condition $u(1)=u(\eta)+1$. It shows the essentiality of condition $S_{1}<1$ for the case $b_{0}=0$. Example 3. The problem

$$
\varepsilon u^{\prime \prime}(x)+u^{\prime}(x)=1,0<x<1, u(0)=0, u(1)=u(\eta)
$$

is ill-posed, it has the unstable on parameter solution

$$
u(x)=-(1-\eta)[\exp (-x / \varepsilon)-1][\exp (-1 / \varepsilon)-\exp (-\eta / \varepsilon)]^{-1}+x,
$$

$u(x) \rightarrow-\infty$ at each nonzero point $x$ if $\varepsilon \rightarrow 0$. It shows the essentiality of condition $S_{1}<1$ for the case if $b_{0}=0$.

Example 4. The problem

$$
\varepsilon u^{\prime \prime}(x)+a u^{\prime}(x)-b u(x)=0,0<x<1, u(0)=0, u(1)=\beta u(\eta)
$$

is ill-posed, in general, for each $\beta>1, \eta \in\left(0, \beta^{-1}\right), a=$ const $>0, b=$ const $\geq 0$. It always has infinite number of solutions for some parameter value $\varepsilon=\varepsilon^{*}$. This fact shows the essentiality of condition $S_{1} \leq 1, i=0,1$. Let us confirm the ill-posedness. (i) If $b=0$, then for an arbitrary constant $C$ the function

$$
u(x)=C \exp (-a x / \varepsilon)-C
$$

satisfies the equation (5.1) and the condition $u(0)=0$. By choosing $C \neq 0$ and substituting (5.2) into nonlocal condition (5.1) we get

$$
\beta=\frac{1-e^{-a / \varepsilon}}{1-e^{-a \eta / \varepsilon}} .
$$

Note, the equality (5.3) is impossible for $-\infty<\beta \leq 1, \eta \in(0,1)$. However, for $\beta>1$ and for each $\eta \in\left(0, \beta^{-1}\right)$ the formula $(5.3)$ is true for some value $\varepsilon=\varepsilon^{*}$. Indeed, for each $\varepsilon>0$ the function $g(\varepsilon)=\left(1-e^{-a / \varepsilon}\right) /\left(1-e^{-a \eta / \varepsilon}\right)$ is positive and continuous, $\lim _{\varepsilon \rightarrow+0} g(\varepsilon)=1$, $\lim _{\varepsilon \rightarrow+\infty} g(\varepsilon)=\eta^{-1}$, the $g(\varepsilon)$ reachs the value $\beta$ at some argument $\varepsilon^{*}$ since $1<\beta<\eta^{-1}$. So, $g\left(\varepsilon^{*}\right)=\beta$, i.e., (5.3) is true for $\varepsilon=\varepsilon^{*}$, therefore, $u(x)$ is solution of (5.1). Since $C \neq 0$ is an arbitrary constant for (5.2), then (5.1) has infinite number of solutions.

(ii) If $b>0$, then for an arbitrary constant $C$ the function

$$
u(x)=C \exp \left(\lambda_{1} x\right)-C \exp \left(\lambda_{2} x\right)
$$

satisfies the equation (5.1) and the condition $u(0)=0$ for

$$
\lambda_{1}=-a / 2 \varepsilon-\sqrt{(a / 2 \varepsilon)^{2}+b / \varepsilon}, \quad \lambda_{2}=-a / 2 \varepsilon+\sqrt{(a / 2 \varepsilon)^{2}+b / \varepsilon} .
$$

By choosing $C \neq 0$ and substituting (5.4) into (5.1) we get

$$
\beta=e^{\lambda_{1}(1-\eta)} \frac{1-e^{\lambda_{2}-\lambda_{1}}}{1-e^{\eta\left(\lambda_{2}-\lambda_{1}\right)}}=\frac{e^{\lambda_{1}}-e^{\lambda_{2}}}{e^{\lambda_{1} \eta}-e^{\lambda_{2} \eta}} .
$$

The equality (5.5) is impossible for $-\infty<\beta \leq 0$. Moreover, (5.5) is impossible for $0<\beta \leq 1$ too. It follows from the relation $h(1)-\beta h(\eta) \neq 0$ for the function $h(t)=e^{\lambda_{1} t}-e^{\lambda_{2} t}, t \in(0,1]$ in view of $h(t)<0, h^{\prime}(t)<0$. However, for $\beta>1$, the formula (5.5) is true for some value $\varepsilon=\varepsilon^{*}$ in respect of each $\eta \in\left(\beta^{-1}, 1\right)$. Indeed, since $z(\varepsilon)=h(1) / h(\eta)$ is positive and continuous function in $(0,+\infty), \lim _{\varepsilon \rightarrow+0} z(\varepsilon)=+\infty, \lim _{\varepsilon \rightarrow+\infty} z(\varepsilon)=\eta^{-1}$, then $z\left(\varepsilon^{*}\right)=\beta$ for some $\varepsilon^{*} \in(0,+\infty)$. So, (5.5) is true for $\varepsilon=\varepsilon^{*}$, then $u(x)$ is solution of (5.1) for 
$\lambda_{i}=\lambda_{i}\left(\varepsilon^{*}\right)$. Since $C \neq 0$ is an arbitrary constant for (5.4), then (5.1) has infinite number of solutions.

\section{Conclusion}

In this article we studied NLBVP of the first kind for linear second order ODE with positive parameter at the highest derivative. We researched the well-posed solvability of the problem in respect of classical solution. Under new and accurate condition on coefficients and nonlocal carriers of NLBVC, we obtained a uniform on parameter stability estimate of classical solution, proved existence and uniqueness. We demonstrated examples of ill-posed problems for some cases if coefficients of NLBVC does not satisfy stated herein well-posedness condition, i.e., we confirmed, in general, that established in our paper condition is essential.

\section{Acknowledgments.}

The author would like to thank Prof. Dr. H. Çakalli which scientific attention to the author's research stimulated to issue this paper result.

\section{REFERENCES}

[1] A. N. Tikhonov, On the dependence of the solutions of differential equations on a small parameter, Mat. Sb., 22(64) (1948), 193-204.

[2] A. V. Bitsadze and A. A. Samarskii, On some simple generalizations of linear elliptic boundary problems, USSR Academy of Science Reports, 185(4) (1969), 739-740.

[3] V. A. Il'in and E. I. Moiseev, A nonlocal boundary value problem of the first kind for the Sturm-Liouville operator in differential and difference interpretations, Differ. Uravn., 23(7) (1987), 1198-1207.

[4] V. A. Il'in and E. I. Moiseev, An a priori estimate for the solution of a problem associated with a nonlocal boundary value problem of the first kind, Differ. Equ., 24(5) (1988), 519-526.

[5] R. Chegis, Numerical solution of problems with small paprameter at higher derivatives and nonlocal conditions, Liet matem. rink., 28(1) 1988, 144-152.

[6] D. M. Dovletov, On the nonlocal boundary value problem of the first kind in differential and difference interpretation, Differ. Equ., 25(8) (1989), 917-924.

[7] D. M. Dovletov, On some nonlocal boundary value problem in differential and difference interpretation, The dissertation ... of the candidate for physical and mathematical sciences, Steklov Mathematical Institute of Russian Academy of Sciences, Moscow (1989), 1-128 (In Russian language: The Russian State Library, The General Digital Catalogue. Website links www.rsl.ru/en, www.rsl.ru : search request for the paper is "Dovletov Dovlet Meydanovich").

[8] D. M. Dovletov, Uniformly difference schemes for nonlocal boundary value problem with a small parameter, "The Differential Equations and Applications". Proceedings of the scientific and practical conference. (Perfomed by The Academy of Sciences of Turkmenistan, Institute of Mechanics and Mathematics, Turkmenistan State University) Ashgabat, Turkmenistan, 1993(2) (1993), 68-72.

[9] N. Adzic, Spectral approximation and nonlocal boundary value problems, Novi Sad J. Math., 30(3) (2000), 1-10.

[10] D. Arslan and M. Cakir, A numerical solution of singularly perturbed convection-diffusion nonlocal boundary problem, Commun. Fac. Sci. Univ. Ank. Ser. A1 Math. Stat., 68(2) (2019), $1482-1491$.

[11] V.A. Il'in and E.I. Moiseev, Second kind nonlocal boundary value problem for Sturm-Liouville operator, Differ. Equ., 23(8) (1987), 1422-1431.

[12] E.P. Doolan, J.J.H. Miller and W.H.A. Schilders, Uniform Numerical Methods for Problems with Initial and Boundary Layers, Dublin, Boole Press (1980).

[13] D.M. Dovletov, Nonlocal boundary value problem in terms of flow for Sturm-Liouville operator in differential and difference statements, e-Journal of Analysis and Applied Mathematics, 2018(1) (2018), 37-55. 
[14] M. Kumar, P. Singh and H.K. Mishra, A recent survey on computational technique for solving singulalrly perturbed boundary value problems, International Journal of Computer Mathematics, 84(10) (2007), 1439-1463.

Dovlet Dovletov,

Near East University, Mersin 10, Turkey. Orcid number:0000-0001-9052-8359 Phone: +99365818716 (Turkmenistan, Ashgabat)

Email address: dovlet.dovletov@gmail.com 